\title{
LITTLE BONEY, GROSSE MENACE : UNE ANALYSE DE L'IMAGE
}

\author{
${ }^{1}$ Witakania SUNDASARI, ${ }^{2}$ Ferli HASANAH \\ ${ }^{1}$ Université de Poitiers, France ; ${ }^{2}$ Universitas Padjadjaran, Indonésie \\ ${ }^{1}$ sundasari.som@univ-poitiers.fr, ${ }^{2}$ ferli.hasanah@unpad.ac.id \\ Article reçu le 13 octobre 2017 | révisé depuis le 16 octobre 2017 | accepté le 24 décembre 2017
}

\begin{abstract}
RÉSUMÉ. Exprimant un message, une image est utilisée comme moyens de communication. Malgré son effet comique, une caricature politique construit indispensablement un discours politique. En 1804 James Gillray a lancé un dessin satirique qui dénonce Napoléon Bonaparte ainsi que les idées républicaines de la Révolution de 1789 pour sensibiliser le public Britannique par ses regards royalistes contre la France révolutionnaire et napoléonienne. La présente recherche s'insère sur l'étude sémiologique de l'image publiée pendant cette période turbulente et l'analyse s'effectue en appliquant les lectures dénotative et connotative sur les signes linguistiques, iconiques et plastiques. Cette analyse a pour but de montrer comment cette caricature communique ses messages et comment l'artiste communique son engagement politique. Les résultats montrent que l'artiste a transmis son message par les parallélismes ainsi que par les oppositions que tous les signes reposent sur l'image.
\end{abstract}

Mots-clés : analyse d'image, angleterre, caricature, révolution française.

\begin{abstract}
Expressing a message, an image is used as a tool of communication. Despite its comic effects, a political caricature unavoidably constructs a political discourse. In 1804, James Gillray launched a satirical drawing that denounced Napoleon Bonaparte and the Republican ideas of the Revolution of 1789 to alert the British public through his royalist point of view against revolutionary and Napoleonic France. This research is based on the semiological study of the image which was published during this turbulent period and the analysis is carried out by applying the denotative and connotative readings on linguistic, iconic and plastic signs. This research aims to show how this caricature transfers its messages and how the artist declared communicate his political commitments. The results show that the artist sent his message through the parallelism as well as the oppositions that all the signs rest on the image.
\end{abstract}

Keywords : caricature, caricature analysis, England, French revolution. 


\section{INTRODUCTION}

The Genius of France Nursing Her Darling est une des caricatures de James Gillray sur laquelle la France est présentée comme une puissance qui menace l'Europe, en particulier, et notamment à la Grande-Bretagne, le pays natal de l'artiste, car La France voulait répandre ses idées révolutionnaires au-delà de ses frontières et lançait des guerres interminables contre les coalitions royalistes européennes. Avec sa politique expansionniste, Napoléon Bonaparte a créé une grande armée en employant une stratégie et une tactique triomphale qui l'ont mené à la victoire. Glorifié et détesté en même temps, Napoléon Bonaparte est sacré ainsi héritier et défenseur de la Révolution. Mais il marqua ironiquement la fin de la Révolution française en se proclamant premier Consul à vie par un coup d'Etat, puis en 1804, en se couronnant Empereur des Français, sous le titre de Napoléon $1^{\mathrm{er}}$.

James Gillray représente le caricaturiste incontestable de la vision anglaise à l'égard de la France révolutionnaire et napoléonienne et " appartient à une première génération d'artistes s'étant entièrement consacrés à la caricature politique » (Porterfield, 2014). Il a témoigné «l'installation des partis politiques modernes en Angleterre sous le règne de George III et, en France, le passage de la Révolution à l'Empire " (Porterfield, 2014) d'où sont venus les sujets hilarants de ses caricatures. Il était tributaire à Golden Age of Caricature au cours duquel la caricature anglaise se développait ayant comme cible particulière la Révolution française :

[l]e bouleversement politique et social, aux enjeux bientôt universels, qui produira la réponse graphique satirique et humoristique la plus violente, la plus grotesque et la plus abondante. Le rire de la caricature anglaise devient alors terrible, à certains égards effrayant et ouvertement anti-français. (Dupuy, 2000)

Issu de l'italien caricatura, la caricature connaît une très longue histoire - la plus ancienne datant 79 av. J-C - dont la diffusion se développe rapidement grâce à l'invention de l'imprimerie. La caricature est définie par Philippe RobertsJones comme

Tout dessin ayant pour but soit de faire rire par la déformation, la disposition ou la manière dont est présenté le sujet, soit d'affirmer une opinion, généralement d'ordre politique ou social, par l'accentuation ou la mise en évidence d'une des caractéristiques ou de l'un des éléments du sujet, sans avoir pour ultime but de de provoquer l'hilarité (cité dans Reshef, 1984)

En fait, «le terme caricature, [...] apparaît lui pour la première fois au milieu du XVIIe siècle » (Dupuy, 2008) et « devient au 18 e siècle l'instrument favori des gravures politiques qui commencent à apparaître à cette époque d'émergence d'une nouvelle réalité qu'on appelle 'l'opinion publique'» (Dupuy, 2000). Quant aux caricatures politiques, «elles, n'hésitent point à s'emparer du sujet afin de couler leur message efficacement dans un moule à la fois familier et provocateur »(Cillessen \& Reichardt, 2010) dont le but est de « déstabiliser par l'arme du rire tout pouvoir trop sûr de lui " (de Baecque, 1997). Par rapport au public, la caricature politique est « une influence évidente, un appel au peuple» (Laurent, 2015) ou «[participe] des combats d'opinion et de propagande et [joue] également le rôle de moyen d'information" (Duprat, 2002). De ce fait, la caricature fournit «des documents très précieux pour l'histoire politique et l'histoire des représentations collectives » (Baridon \& Guédron, 2011).

La caricature est une dessin de l'humour noir et sarcastique où «la représentation [...] d'une personne ou [...] d'un sujet, dans laquelle la vérité et la ressemblance exacte ne sont altérées que par l'excès du ridicule » (cité par Duprat, 1999). Sur l'image, l'artiste met souvent des choses dégradantes, grotesques, grivoises, même brutales ou violentes. «Dessin polémique, la caricature, si elle n'a pas toujours vocation à déclencher le rire, cherche nécessairement à ridiculiser, à choquer, à provoquer, ou encore à stigmatiser une situation ou une 
personne » (Duprat, 1999). Les dessins satiriques décrivent audacieusement avec des «déformations physiques des personnages » (Baridon \& Guédron, 2011) ou sous formes variés: zoomorphisation ou animalisation, transformations en êtres oniriques ou fantasmatiques. Selon Bertrand Tillier, le corps caricaturé est " comme un lieu d'un contre-pouvoir et comme la métaphore du régime républicain désacralisé » (cité par Bonhomme, 2010).

Devant une œuvre, plusieurs lectures sont possibles. Certains chercheurs relient le contexte à la compréhension des œuvres, alors que d'autres mettent en avance la vie de l'artiste et son parcours. Pour interpréter, certains utilisent la psychanalyse tandis que d'autres la sémiologie. La recherche de Hildegard Meister ((Meister, 1993) montre que la caricature se fonctionne comme moyens de communication voire comme un discours politique de l'artiste. Cette recherche oriente notre recherche dont l'objet d'étude de cette analyse est la caricature The Genius of France Nursing Her Darling de James Gillray. Le choix est basé premièrement sur le fait que cette caricature, crée en 1804, est représentatif des périodes turbulentes dans l'Histoire de la France : La Révolution de 1789 qui a mis fin l'Ancien Régime et qui a été mis fin à son tour par le couronnement de l'empereur. Deuxièmement, sachant que James Gillray est renommé l'un des plus grands caricaturistes Britanniques, cette caricature nous a incité une curiosité sur le regard des Britanniques - représenté par l'artiste - à l'encontre de la France, dont la rivalité de ces deux pays s'est écrite dans la connaissance commune. De ceci, par cette présente recherche, nous tentons de faire montrer ce qu'une caricature communique ses messages ainsi que ce que l'artiste communique ses engagements politiques.

Par la ressemblance aux objets qu'elle décrit, une image est considérée lisible naturellement. Pourtant, reconnaître les personnages et les objets présents sur la scène ne signifie pas que l'on comprenne le message de l'image. Cela amène à la nécessité d'un regard analytique et en profondeur pour pouvoir «lire» et comprendre mieux la signification de l'image et ensuite ses messages. De ceci, cette analyse a pour problématique : (1) de quelle manière l'image communique-t-elle des messages ?; et (2) comment l'artiste fait-il comprendre le monde à travers ses yeux?

Apparemment naïve et naturelle, la caricature est en effet secrètement codée, d'où l'objective de cette analyse: la compréhension en profondeur de l'image en profitant les connaissances initiales de significations pour ensuite dévoiler le message de l'image. Pour mieux comprendre la caricature et sa spécificité sous l'angle de la signification, la théorie sémiotique de l'image de Roland Barthes est nécessaire à appliquer. Chaque chose désigne ou signifie autre chose. Selon Ferdinand de Saussure, chaque mot comme un signe linguistique - relie un signifiant (= le son) à un signifié (= le concept), tandis que, dans une perspective plus large, Charles Sanders Pierce explique qu'un signe montre une relation entre un representamen (= signifiant), un objet (= référent) qu'il représente, et un interprétant (= signifié) qu'il signifie (dans Joly, 1994). Les différents types de relations tripolaires distinguent le type de signes: une icône correspond à une relation analogique entre le signifiant et son référent; un indice à une relation causale; tandis qu'un symbole à une relation conventionnelle (Joly, 1994).

La sémiologie de l'image permet «d'aborder l'image sous l'angle de la signification » (Joly, 1994) pour ensuite l'interpréter. Relier ou associer un signifiant (l'image et ses objets) à un signifié (le concept) pour une interprétation. L'image est considérée comme un signe et donc constitue des messages. Par l'interaction entre les signes iconiques et les signes plastiques et parfois les signes linguistiques, l'image nécessite à décrypter afin de la comprendre.

Outre, pour comprendre une image dont une caricature, une connaissance de 
la signification conventionnelle est importante, aussi bien de la façon de dessiner qui «respecte des règles représentatives convenues » (Joly, 1994) tels que les règles de la perspective ou celles des tiers. En utilisant des lignes imaginaires, la règle des tiers divise une image « en 9 rectangles égaux en traçant 2 lignes horizontales et 2 lignes verticales sur les tiers » (Beilhe, 2012) afin de positionner les points d'intérêt de l'image le long de ces ligne ou à leur point d'intersection. Les lignes horizontales et verticales sont nommées les lignes de force, les points aux intersections composés par les lignes de force sont nommés les points forts.

S'appuyant sur la théorie de la sémiologie de l'image, cette recherche va montrer comment la caricature de James Gillray The Genius of French Nursing Her Darling communique ses messages qui représentent à son tour les engagements politiques de l'artiste.

\section{MÉTHODE}

La recherche que nous menons porte sur une caricature de James Gillray (1756-1815), intitulée The Genius of French Nursing Her Darling, qui a été publié en 1804 (voir figure 1). Nous analysons cette image en appuyant sur la théorie de Roland Barthes considérant une image comme phénomène sociologique vu $\mathrm{qu}^{\prime}$ elle produit du sens. Pour faire une analyse de l'image, il introduit deux niveaux de lectures: une lecture dénotative pour le sens premier et une lecture connotative pour le deuxième (dans Joly, 1994). Cette analyse applique la même méthode: par une lecture dénotative au premier plan et ensuite par une lecture connotative au deuxième.

L'analyse est commencée par une lecture dénotative au cours de laquelle nous décrivons des objets trouvés sur scène et leurs significations basées sur la connaissance commune ou sur la convention. Après, une lecture connotative qui est interprétative est appliquée sur ces significations plus profondes ayant pour but de comprendre le message de l'artiste concernant ses regards sur l'événement parlant.

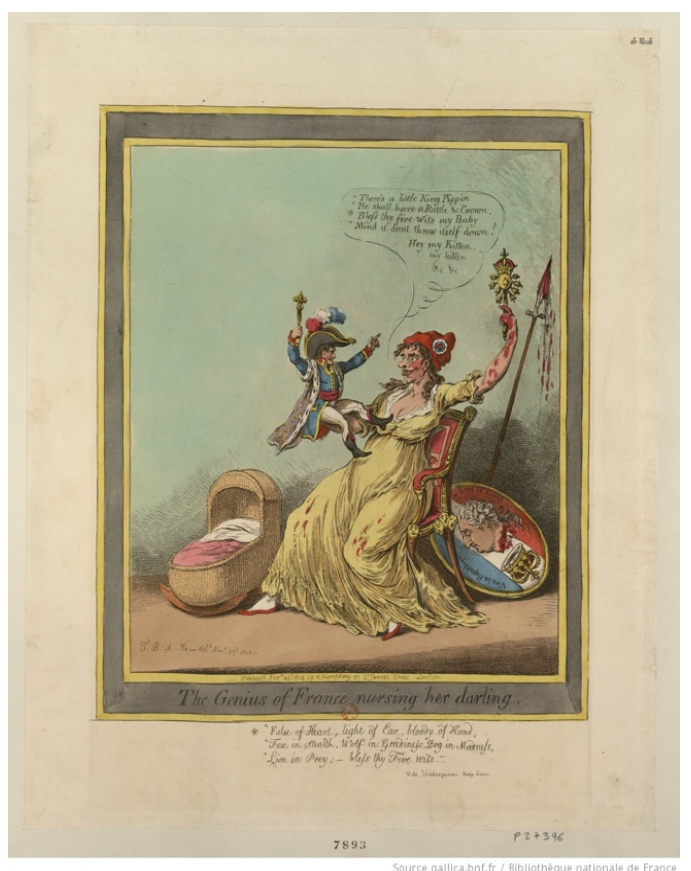

Figure 1. The Genius of France Nursing Her Darling, 1804, eau-forte colorisée, $354 \times 260 \mathrm{~cm}$ («The Genius of France Nursing Her Darling », s. d.)

\section{RÉSULTATS ET DISCUSSION}

Pour analyser la caricature The Genius of France Nursing Her Darling, deux niveaux de lectures s'effectuent respectivement: lecture dénotative et lecture connotative.

\section{Lecture dénotative}

Pour comprendre le message de la caricature analysée, il faut d'abord trouver des signifiants qui vont être reliés aux signifiés. Pour parler de la France révolutionnaire et napoléonienne, l'artiste désigne les objets liés à l'idée de la France en tant que tels. Au premier niveau, la lecture se fait par les trois signes tels que signes linguistiques, signes iconiques et signes plastiques.

\section{Signe linguistique}

Le titre de la caricature est bien marqué et se trouve sur l'encadrement de l'image dont le mot «nursing » amène à l'idée de la relation mère-enfant. Ce signifiant va avec une soi-disant comptine: 
There's a little King Pippin

He shall have a Rattle \& Crown

*Bless thy five wits my Baby

Mind it don't throw itself down!

Hey my Kitten

my kitten..

[Il y a un petit Roi Bien-Admiré

Il aura un hochet et une couronne

* Bénis tes cinq esprits mon bébé

Attention, ne te jette pas !

Salut mon chaton

mon chaton... ]

En chantant une comptine, la mère berce son enfant et l'entoure de l'amour et de l'admiration. Elle cite également ce qu'elle espère le meilleur pour son bien aimé.

Sur le bouclier s'écrit 'Vive la République' et 'the Last of King' comme slogan de la Révolution française de 1789.

\section{Signe iconique}

Le signifiant de la présence mèreenfant est aussi représenté par les signes iconiques. Par la posture des personnages sur la scène fabriquant une photo d'une mère et son petit bébé. Cette impression est renforcée par la présence des objets socioculturels déterminés: un berceau « symbole du sein maternel et de la protection de la petite enfance» (Oesterreicher-Mollwo, 1992) qui se trouve au sol, tout près des pieds de la femme, deux « hochets »; l'un est tenu par la mère et l'autre par l'enfant que décrit l'artiste comme un enfant admiré (pippin), ayant encore envie d'un hochet bien qu'il en a déjà un.

Outre, sur la scène, il y a aussi les signifiants qui renvoient à l'idée de la France que l'on peut catégoriser en trois périodes de l'Histoire de la France, à savoir respectivement l'Ancien Régime, La Révolution française de 1789 et la République, et la France sous l'empereur Napoléon le $1^{\mathrm{er}}$. Les sceptres - l'un surmonté d'une fleur de lys et l'autre d'une croix - la couronne et la trône, l'hermine et la fourrure, tous signifient la France de l'Ancien Régime, ainsi que (la tête de) Louis XVI le dernier roi. Le bonnet phrygien et la cocarde et aussi Marianne sont les signifiants de la Révolution française et la République. Alors que Napoléon devient lui le signe iconique de la dernière période.

\section{Signe plastique}

Les couleurs les plus présentes sur la scène sont le bleu, le blanc et le rouge qui évoquent tous les trois les couleurs du drapeau français, officiellement établie en 1958 par la Constitution. Or, ces trois couleurs sont connues depuis longtemps. Bien souvent que l'on les associées à la Révolution française de 1789, en fait « [la] monarchie française sur le point de se consolider, ce sont ses couleurs qui deviendraient d'abord celles du Royaume de France »(Bertozzo, 2016). Le tricolore est donc un signifiant de l'État français.

En somme, tous les signifiants de ces trois types de signes présentent sur l'image renvoient «naturellement» à l'idée de la France, voire à ce qui s'est passé à un moment donné en France. Par une bonne reconnaissance des significations voire des objets associés à la France, cette lecture dénotative nous amène à un contexte historique dont parle l'artiste.

\section{Lecture connotative}

Après la lecture dénotative, une lecture connotative se fait pour que l'on arrive à une interprétation en profondeur de l'image.

\section{Signe linguistique}

Si par la lecture dénotative, nous avons remarqué une soi-disant comptine nous amenant à l'idée de la relation mèreenfant, où la mère berce son enfant en lui chantant ses espérances pour son bien aimé.

*Bless thy five wits my Baby

Cette phrase est inspirée de la pièce King Lear de William Shakespeare où sur acte 3 scène 4, lorsque Edgar se retrouve avec King Lear. Edgar dit "Bless thy five wits, Tom's a-cold" ainsi sur acte 3 scène 6 «Bless thy five wits» (Walthall, 2016). Edgar est le fils légitime du comte de 
Gloucester. Il doit s'enfuir en se déguisant en Tom le mendiant à cause de l'ambition et de la trahison d'Edmond -le fils illégitime de son père - qui convoite l'héritage du comte en faisant tournant le dernier contre Edgar. Alors que King Lear s'amène à un destin tragique par sa fierté mais aussi par la trahison de ses deux filles et de ses gendres. Cette tragédie parle «de la folie et de la manipulation, les conséquences de la cupidité [...] un avertissement simple sur ce qui se passe lorsque nous comptons sur notre fierté et notre égo, plutôt que notre raison, pour prendre nos décisions » (Littlehale, 2017). De ceci, nous pouvons lier le thème de la pièce au contexte à un moment donné de la France.

King Lear is about political authority as much as it is about family dynamics. Lear is not only a father but also a king, and when he gives away his authority to the unworthy and evil Goneril and Regan, he delivers not only himself and his family but all of Britain into chaos and cruelty. («SparkNotes: King Lear: Themes, Motifs \& Symbols », s. d.)

[Roi Lear parle autant de l'autorité politique que de la dynamique familiale. Lear est non seulement un père mais aussi un roi, et quand il cède son autorité à l'indigne et au mal Goneril et Regan, il livre non seulement lui-même et sa famille, mais toute la Grande-Bretagne dans le chaos et la cruauté.]

Pareillement à King Lear, le roi Louis XVI a perdu son pouvoir lorsqu'il le «lègue» à sa fille Marianne (à lire la République française) qui ensuite le « lègue » à son tour à Napoléon d'où le chaos et la violence qui règnent en France ainsi qu'en Europe.

Cependant, les «five wits » nous font penser à cinq sens : ouie, vue, odorat, goût et touche. Alors que cet écrivain anglais parle plutôt de: common wit, imagination, fantasy, estimation, et memory (Walthall, 2016). Sur cet acte, le personnage Edgar s'assume sincèrement ses défauts et ses erreurs devant King Lear devenu fou :

A servingman, proud in heart and mind, that curled my hair, wore gloves in my cap, served the lust of my mistress' heart and did the act of darkness with her, swore as many oaths as I spake words and broke them in the sweet face of heaven - one that slept in the contriving of lust and waked to do it. Wine loved I deeply, dice dearly, and in woman outparamoured the Turk. False of heart, light of ear, bloody of hand - hog in sloth, fox in stealth, wolf in greediness, dog in madness, lion in prey. Let no creaking of shoes nor the rustling of silks betray thy poor heart to woman. Keep thy foot out of brothel, thy hand out of plackets, thy pen from lender's books, and defy the foul fiend. («No Fear Shakespeare: King Lear: Act 3, Scene 4, Page 4 », s. d.)

[Un serviteur, fier de cœur et d'esprit, qui me bouclait les cheveux, portait des gants dans ma casquette, servait la convoitise du cœur de ma maîtresse et faisait l'obscurité avec elle, jurait autant de jurons que je prononçais des mots et les cassait dans la douce face du ciel - une qui a dormi dans le désir de la convoitise et s'est réveillé pour le faire. Le vin que J'aimais profondément, découpait chèrement, et chez la femme, il excitait le Turc. Faux de cœur, lumière d'oreille, sanglant de main - sanglier en paresse, renard furtif, loup en avidité, chien en folie, lion en proie. Que le grincement des souliers et le craquement des soies ne trahissent pas ton pauvre cœur à la femme. Garde ton pied hors du bordel, ta main hors des pattes, ta plume des livres du prêteur, et défie du démon.]

Faisant référence explicitement à King Lear de Shakespeare, Gillray compare la France révolutionnaire et napoléonienne à la tragédie puisque «all the values that we think of as protecting our sense of humanity are attacked» (Woods, 2016). James Gillray écrit sur cette caricature :

* "False of Heart, light of Ear, bloody of Hand,

"Fox in stealth, Wolf in Greediness, Dog in Madness,

"Lion in Prey; - Bless thy Five Wits" vide Shakespeare's King Lear

[Faux de cœur, lumière d'oreille, sanglante de main,

"Fox en furtivité, Loup dans la gourmandise, Chien dans la folie, 
"Lion à Proie ; - Bénis tes Cinq Esprits

"voir le Roi Lear de Shakespeare]

Nous remarquons ici une modification de ce qu'écrit Shakespeare. Gillray ne mentionne pas «hog in sloth» qu'il remplace, par contre, par "false of heart, light of ear, bloody of hand ». Au lieu de parler des cinq vertus - à savoir les « five wits", il aborde les défauts pour dénoncer Bonaparte par la mise en œuvre " the madness », une ressemblance entre le roi Lear et l'empereur Napoléon $1{ }^{\mathrm{er}}$.

Par ces signes linguistiques qui relient la tragédie à un moment donné en France, nous pouvons donc interpréter un parallélisme entre ces deux «histoires »: la trahison qui évoque une situation chaotique suite d'un coup de pouvoir. Cette idée est renforcée par d'autres signes linguistiques «vive la République» et «The last king». Or, la phrase «Vive la République » devient une moquerie car la République elle-même a été remplacée ironiquement en empire par Napoléon Bonaparte lorsqu'il se nomme Empereur des Français. C'est de la trahison des idées révolutionnaires et républicaines. Or, la phrase «Vive la République » devient une moquerie car la République elle-même a été remplacée ironiquement en empire par Napoléon Bonaparte lorsqu'il se nomme Empereur des Français. C'est de la trahison des idées révolutionnaires et républicaines. Bref, il existe clairement ici une double trahison, juste comme ce qu'écrit Shakespeare dans sa pièce King Lear.

\section{Signe iconique}

Parmi les objets sur la scène, certains renvoient à l'idée du royaume de France. «Comme le pouvoir royal doit être identifié pour être reconnu comme tel par l'ensemble du royaume» (Duprat, 2002) d'où la création des objets emblématiques. Ce ne sont pas les objets quelconques, mais représentateurs du pouvoir de la monarchie: une couronne comme «ornement destiné à magnifier celui qui le porte » (Rapelli, 2005) ou un sceptre comme "symbole de la puissance et de la dignité suprême, souvent considéré comme porteur de la force royale» (Oesterreicher-Mollwo, 1992). Surmonté d'un globe comme symbole du monde, un sceptre symbolise également « du pouvoir absolu sur ce dernier» (Rapelli, 2005).

Outre ces emblèmes, la monarchie se montre par sa grandeur représentée par l'habillement. Selon le dictionnaires Symboles du pouvoir (Rapelli, 2005) une hermine «symbolise la dignité, l'honneur et le pouvoir de rendre la justice » dont les fourrures symbolisent ainsi "préséance, pureté de mœurs, doctrine, religion, maîtrise et autorité [...] de sagesse supérieure, d'autorité innée, la garantie d'une éthique supérieure, super partes ». En France, l'hermine du roi est souvent décorée des fleurs de lys comme «un symbole de pureté, propre aux armes des Capétiens et des dynasties françaises successives. " La fleur de lys est aussi présente sur d'autres emblèmes du royaume tels que sceptre ou trône.

Par opposition à la grandeur du royaume, il existe des objets populaires signifiant la Révolution et, par la suite, la République. Inspirée du bonnet daté de l'Antiquité portant par des esclaves, pendant la période de la Révolution de 1789, le bonnet phrygien devient un symbole «de la souveraineté populaire» («Les symboles de la Révolution - La Révolution Française», s.d.). Le bonnet phrygien est souvent décoré d'une cocarde tricolore, le symbole de la Révolution.

De tradition judéo-chrétienne, les rois du royaume « ont été hantés par l'idée de persuader les peuples de leur légitime, une légitimité transcendante placée à la fois sous le signe de Dieu, grâce à l'onction $\mathrm{du}$ sacré, et du respect des lois fondamentales du royaume. » (Duprat, 2002). La mise en compte ce pouvoir divine est représentée par exemple par la croix du Christ comme «signification symbolique de la Passion et du triomphe du Christ »(Oesterreicher-Mollwo, 1992). Le croix du Christ est donc présente sur le trône symbolisant «d'unité stable, de synthèse entre ciel et terre » (Rapelli, 2005), ou sur le sceptre comme «attribut 
fréquent des divinités » (OesterreicherMollwo, 1992) qui symbolise «la protection $\mathrm{du}$ 'troupeau' des fidèles ou, dans un cadre non religieux, de la masse des sujets » (Rapelli, 2005).

L'idée de la protection est renforcée par un autre objet: le bouclier. Cet arme défensive est néanmoins en contradiction avec la lance qui, étant une arme offensive, «symbolise la guerre» (OesterreicherMollwo, 1992). Par la mise en image d'un bouclier, au sol, à côté d'une lance, debout et ensanglantée, l'artiste a montré que la justice, la prudence et la protection symbolisée par le bouclier - sont défaites par le guerrier - symbolisé par la lance. Cette idée de défaite est ainsi représentée par ce qui est décrit sur le bouclier : sur lequel repose la tête désincarnée sanglante du roi Louis XVI et sa couronne renversée. L'artiste dessine ici un roi qui a été exécuté, ainsi qu'un coup de pouvoir contre le royaume lors des chaos connut la France révolutionnaire. La Révolution qui a mis fin au royaume de France est symbolisée par la tête et la couronne renversée du le dernier roi de l'Ancien Régime, «Le malheureux Louis XVI enfin, qualifié par les royalistes eux-mêmes de 'roi de pétaud', ou encore de 'roi zéro', est également transformé par les patriotes, tenants de la Révolution, en roi cochon » (Duprat, 1999).

Sur ces deux derniers objets, nous remarquons une autre signifiante bien présente : le sang. "Dans le christianisme, le sang est interprété comme une force expiatoire et libératrice ", mais paradoxalement, le sang signifie aussi l'impureté (Oesterreicher-Mollwo, 1992).. Le sang se montre ainsi comme des taches sur la robe de Marianne et sur sa main gauche.

Une caricature est toujours une exagération de quelque chose :

Dessin polémique, la caricature ne cherche pas toujours à déclencher le rire, mais elle déforme, parodie, raille, ridiculise, dénoncé une situation ou le comportement d'une personne ou d'un groupe social. Ses trois fonctions de base sont : exagérer, défigurer, accuser. Elle vise donc à mettre en évidence divers caractères physiques ou moraux de personnages et à toucher efficacement ses spectateurs, grâce à l'accentuation du trait et à sa force de simplification. C'est un art de la subversion qui déforme le modèle, s'attaque à la personne publique, à son image, à ses sentiments, à sa politique, en faisant preuve d'un irrespect sans limite. (Salles \& Eymard, s. d.)

Sur la scène, contrairement à l'image commune de Marianne comme une jolie jeune femme fine, Gillray dénigre Marianne comme une grande femme grassouillette aux mains rondelettes. Elle a un gros nez, une grande bouche avec des dents proéminentes. Ses gros yeux sont grand ouverts sous ses sourcils épais. Sa taille imposante renforce les traits de caractère d'une femme diabolique. Tandis que pour dessiner Napoléon Bonaparte, l'artiste utilise odieusement le nanisme, c'est-à-dire l'art de minimiser son personnage à la taille lilliputienne, d'où l'artiste le rebaptise « Little Boney » depuis 1803. D'une manière opposée au portrait officiel voire idéalisé de l'Empereur, James Gillray le déformé avec une forte dépréciation : «[Courbe] le menton de son modèle et lui étire la bouche en un trait amer. Ses maxillaires s'allongent pour mieux dépeindre sa bestialité tandis que son allure chétive indique par défaut son appétit démesuré » (Wacheux, 2009).

Malgré ces exagérations, les personnages sont quand même reconnus par exemple par leurs icones vestimentaires. A part son uniforme militaire, Napoléon Bonaparte est facilement reconnaissable à son chapeau surnommé le 'Petit chapeau', qui est devenu l'un des symboles qui caractérise sa silhouette. " Dans l'imaginaire collectif, Napoléon et son chapeau ne font qu'un» (Huguenaud, 2002). Pareillement, Marianne elle est aussi reconnue par son bonnet phrygien, décoré d'une cocarde.

Par ces signes iconiques que nous venons d'analyser, encore une fois nous remarquons que l'artiste continue à mettre en opposition un objet contre 
l'autre ainsi qu'un personnage contre l'autre.

\section{Signe plastique}

Pour montrer l'importance du personnage, James Gillray applique ici la règle des tiers comme suivant :

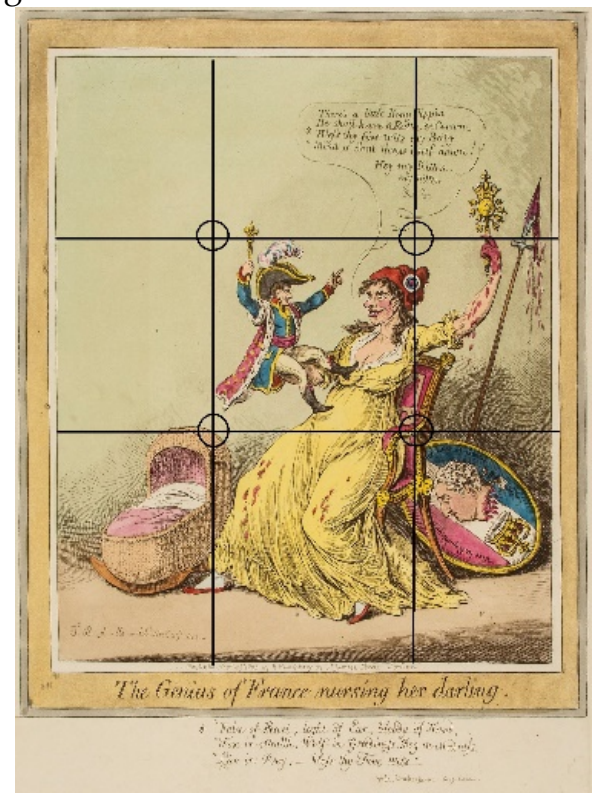

Figure 2. Règle des tiers

Sur la scène, tous les personnages et les objets sont placés sur les cadres gauches bas en laissant le vide à l'intersection gauche supérieure qui représente «en occident l'angoisse ou la perte » («Reflexion sur la notion de vide dans la peinture», s. d.). Quant à la composition, les deux personnages sont encadrés par les points forts. Suite logique, Marianne et Napoléon Bonaparte occupent le centre du tableau et tiennent donc le rôle des personnages principaux.

La caricature se compose de deux plans: tous ceux qui représentent la France révolutionnaire et napoléonienne sont au premier plan, du côté gauche, tandis que ceux qui représentent l'Ancien Régime sont au deuxième plan, du côté droite. Cette mise en place montre comment le premier fait tomber ceux du deuxième ainsi qu'ils les cachent derrière le trône sur lequel Marianne est assise. Assise sur le trône avec un sourire malin, Marianne porte son enfant chéri par sa main droite et la main gauche dégoulinante de sang tient un sceptre surmonté d'une croix qu'elle agite comme un hochet en chantant une comptine.

Porté dans la main de Marianne, Napoléon a son index pointé vers ce sceptre plus grand que celui qu'il tient par sa main gauche. Ce n'est pas un geste anodin car chaque geste transmet un message : " l'index pointé est la trace d'un discours d'ordre» (Arasse, 1981). Et lorsque l'index est pointé vers haut, «il traduit la volonté d'un pouvoir supérieur ou signifie que ce pouvoir est défie » (Pasquinelli, 2006). De ce geste simple, l'artiste a une forte intention de démontrer les désir de toute-puissance de Napoléon Bonaparte ou de «folie de grandeur» (Brown, 2007).

Etant un enfant roi, l'Empereur Napoléon Ier règne sur la France. Pourtant, ces deux personnages Marianne et Napoléon - se regardent dans les yeux. C'est un signe d'échange ou de complicité. Cependant, la façon de mettre en place d'un personnage a aussi une signification: «[Comme] les empereurs qui l'ont précédé, Napoléon n'effleure pas directement la terre de ses pieds. Il se tient à une certaine hauteur, comme il sied à l'autorité éternelle et immuable. » (Rapelli, 2005)

Il en va de même avec le choix de couleurs de cette caricature : rouge, jaune, bleu, blanc et même noir. Bien que les significations que revêtent les couleurs sont différentes selon les pays, les cultures et les époques, mais «[les] couleurs ne sont pas anodines. Elles véhiculent des codes, des tabous, des préjugés auxquels nous obéissons sans le savoir; elles influencent notre environnement, nos comportements, notre langage et notre imaginaire » («Les couleurs », s. d.).

Selon Petit dictionnaire de symboles (Oesterreicher-Mollwo, 1992), étant couleur du sang, le rouge est une couleur symboliquement paradoxale : " couleur de la vie, de l'amour, de la chaleur, de la passion, de la fertilité » mais aussi «celle de la guerre, de la puissance destructrice du feu, du sang répandu, de la haine ». Sur la caricature que nous analysons, le rouge symbolise plutôt le sens négatif de 
cette couleur puisque cette couleur est présente sous forme de taches de sang: sur le bouclier et la lance, ainsi que sur la robe et la main de Marianne. Cela nous renvoie à l'horreur brute, au meurtrie.

Cependant, dans la culture romaine, le rouge est « la couleur du pouvoir, celle de l'empereur, de la noblesse et des généraux » et «[les] autorités judiciaires portent volontiers du rouge, de même que le bourreau, seigneur de la vie et de la mort » ou encore «Satan, le seigneur des enfers, et Babylone la prostituée en sont tout autant revêtus, expression de la force dévastatrice du feu de l'enfer et des appétits insatiables de la passion ». Coiffée d'un bonnet rouge et portant des chaussures de la même couleur, nous ne pouvons pas ne pas associer Marianne à cette dernière signification $\mathrm{du}$ rouge. Empereur, Napoléon est vêtu de l'hermine rouge mettant en cachette son uniforme militaire : un manteau bleu à larges revers jaunes et rouges; une culotte blanche couronné de ruban rouge; et aussi des souliers pointus noirs. Un soldat se déguise en roi.

La couleur de son uniforme est une « couleur du divin, de la vérité, et par le biais du maintien de la vérité et de la permanence du firmament, couleur de la fidélité. C'est aussi la couleur de l'irréel, $\mathrm{du}$ fantastique» ou encore «perdre la raison à cause de l'ivresse ». Par ce choix très recherché de couleurs de vêtements, l'artiste a pour but de montrer une image démoniaque de la France. En laissant Napoléon se sacrer l'Empereur, la France trahit ses idées républicaines voire trahit sa Révolution et son peuple en rejetant la faute sur la République pour tous les chaos et les violences.

Outre le rouge, le jaune est également des significations paradoxales. C'est une "couleur claire, associée à la symbolique de l'or, de la lumière et du soleil » mais aussi « mauvais, diabolique ». Dans la culture romaine, c'est une couleur " réservée aux prêtres et aux gouvernants ; elle devint pour cela symbole de la puissance et de la dignité ", paradoxalement c'est aussi une couleur «du vêtement d'opprobre des Juifs, des hérétiques et des prostituées, d'autant plus que depuis le Moyen Age, faire portant quelqu'un d'un habit jaune, c'est une façon dévaloriser. En Occident, la couleur jaune est d'ailleurs un symbole «de la trahison, de la tromperie et du mensonge » (Simonnet, 2004). Sur la caricature, l'artiste désigne intentionnellement Marianne en robe jaune.

The Genius of France Nursing Her Darling est une gravure en couleur. Parmi les couleurs qu'utilise l'artiste, le rouge et le jaune sont les plus remarquables. Ces deux couleurs sont considérées comme des couleurs chaudes, avec une certaine signification: le rouge est associé au feu mais aussi à la violence et au danger, alors que le jaune qui est associée à la tromperie, la lâcheté et aussi au danger (Chapman, 2014). L'artiste travaille sur son choix de couleurs (à lire les couleurs de ton chaude) pour renforcer le sentiment d'angoisse ou d'inquiétude provoqués par le danger qu'incarne la France. Ceci est renforcé par la présence de la couleur noire signifiant «de l'indifférence, de l'insondable, pour exprimer l'obscurité, le chaos primordial, la mort. Couleur du deuil [...], il est signe d'affliction dénuée d'espoir. » (Oesterreicher-Mollwo, 1992).

$\mathrm{Au}$ niveau de la lumière, elle vient du haut, du côté gauche. Cela dit du côté où l'artiste a mis son personnage principal, dont l'ombre couvre un petit peu Marianne; puis Marianne et Napoléon font de l'ombre au portrait de Louis XVI. Voire ils le cachent derrière eux. Il est d'ailleurs englobé par des nuages noircis, « la couleur du mal» (OesterreicherMollwo, 1992). Gillray met sur la scène l'histoire noire de la France. D'une part il y a la victoire de l'Empereur Napoléon Ier. D'autre part il y a également les violences et la trahison. De ce fait, il existe de l'angoisse, de la peur et aussi de la haine.

En somme, The Genius of France Nursing Her Darling avec ses traits défavorisant et des connotations péjoratives influence le regard du public anglais à l'encontre du nouveau régime français. L'Empire français est une 
trahison contre ses valeurs et ses idées révolutionnaires qui s'étaient auparavant appliquées par des violences de masse. Gillray a fait passer ses reproches contre la France et Napoléon Bonaparte, sa cible primaire. Il a essayé de sensibiliser son public aux dangers qui menacent la Grande-Bretagne : la paix qui s'éloigne, les guerres qui se répandent dans toute l'Europe, la possibilité d'embarquement des armées françaises en Angleterre. Par cette œuvre, Gillray affirme la peur et l'angoisse croissance des Britanniques de l'invasion par Napoléon Bonaparte de la Grande-Bretagne. Il y a vraiment de quoi s'inquiéter et avoir peur vis-à-vis la politique expansionniste et conquérante de la France.

Cette satire graphique qui montre l'image diabolique de la France révolutionnaire et napoléonienne dévoile un message de l'artiste convié par son engagement politique. Confident $\mathrm{du}$ patriotisme et du conservatisme, James Gillray est connu pour sa vision antirépublicaine et antijacobine. Il est également contre - en terme utilisé par George Sackville Germain - « ces maudits Français » (Cillessen \& Reichardt, 2010) et contre tous ceux qui, en Angleterre, osent diffuser des opinions radicales ou révolutionnaires. "He tackled the furies of the French Revolution - followed by Britain's long war against the French»(Rose, 2015). Gallophobe, James Gillray est connu également pour «ses attaques contre la France révolutionnaire et napoléonienne » dont Napoléon Bonaparte comme «un cachet humiliant qui lui tiendrait désormais lieu d'emblème ridicule, récupéré par d'autres caricaturistes continentaux ou britanniques » (Porterfield, 2014). Il a discrédité sans conteste l'image de la France en lançant ses regards anti-français. Grâce à la liberté d'expression quasi-totale en Angleterre, il utilisait la caricature comme "outil de débat intérieur dans le conflit qui oppose son pays à la France, personnalisée par Napoléon Bonaparte » (Ouvrand, 2007).

$\mathrm{Au}$ niveau esthétique, cette caricature a intelligemment réussi à faire reconnaître les personnages et a décrit clairement son contexte historique. Les explications ne sont pas donc nécessaires. Caricaturiste pareillement graveur, les idées politiques de Gillray sont bien traduites par cette caricature analysée. Par son image épouvantable, l'artiste est bien arrivé à transmettre son message : la peur du peuple Britannique de l'invasion par la France révolutionnaire et napoléonienne. Devant l'ampleur de ce qui s'est passé entre ces deux pays, sa critique, malgré sa violence, reste raisonnable et acceptable. Certes, la guerre et les luttes pour le pouvoir restent toujours un grand thème universel abordé par les artistes à travers leur travail.

Marie-Laure Wacheux (2000) considère que les œuvres de James Gillray représentent « la qualité informative de ses gravures révèle un long et patient travail de recherches". Il est sans doute un dessinateur indissociable de la caricature anglaise. The France Genius Nursing Her Darling renforce l'association grotesque de la silhouette caricaturale et iconique de 'Little Boney' à ses caractères démoniques. Depuis sa création en 1803, l'image 'Little Boney' s'est imposée dans l'imaginaire populaire britannique et le reste encore aujourd'hui. Selon Francisque Sarcey (cité dans Reshef, 1984) «La caricature grave [dans la mémoire] des images dont les formes et les couleurs flottent dans le souvenir longtemps encore après qu'on les a vues ».

\section{CONCLUSION}

Par les deux niveaux de lecture, nous pouvons conclure que la caricature communique ses messages à travers des objets qui sont associés étroitement à l'image de la France révolutionnaire et napoléonienne que dénonce l'artiste. Ces objets présentent les parallélismes et les oppositions où toute la dignité et la grandeur de la France sont mise côte à côte avec la grossièreté et la médiocrité pourvu que l'artiste ridiculise l'État qu'il déteste tant. Du portrait de la Révolution française de 1789 et de l'incarnation de propagande de Napoléon, James Gillray dévoile les 
images diaboliques de la France révolutionnaire et napoléonienne pour ensuite transférer un message discréditant la France et Napoléon Bonaparte.

L'artiste se forge une image démoniaque de la France et surtout de Napoléon Bonaparte faite de son engagement politique royaliste pour faire comprendre cette période turbulente de l'histoire de la France en pointant du doigt à la Révolution et à la trahison par la mise en évidence des oppositions et des paradoxes représentées par les signes linguistiques, les signes iconiques et les signes plastiques.

\section{REMERCIEMENTS}

Nous adressons nos remerciements aux personnes qui nous ont aidées dans la réalisation de cet article. En premier lieu, nous remercions Mme. Anne-Cécile GUILBARD, maître de conférences en Littérature française et Esthétique de l'image de l'Université de Poitiers. En tant qu'enseignante de cours de L'image politique, elle nous a guidées dans notre travail. Nous remercions aussi M. Yves LETEURTRE pour sa précieuse aide à relecture et à la correction de cet article.

\section{RÉFÉRENCES}

Arasse, D. (1981). L'index de Michel-Ange. Communications, 34(1), 6-24. https://doi.org/10.3406/comm.1981.1 505

Baridon, L., \& Guédron, M. (2011). Caricaturer l'art : usages et fonctions de la parodie. In S. Le Men (Éd.), L'art de la caricature (p. 87-108). Paris: Presses universitaires de Paris Ouest. Repéré à http://books.openedition.org/pupo/2 216?lang=fr

Beilhe, F. (2012). La règle des tiers. Repéré à https://www.astuces-photo.com/laregle-des-tiers/

Bertozzo, M. (2016). Le 15 février 1794, la Révolution française affiche ses couleurs: De l'adoption du drapeau tricolore. Repéré à à l'adresse http://www.revuegeneraledudroit.e u/blog/2016/02/22/15-fevrier-1794- la-revolution-francaise-affiche-sescouleurs/

Bonhomme, M. (2010). La caricature politique. Mots. Les langages $d u$ politique, (94), 39-45. https://doi.org/10.4000/mots.19858

Brown, H. G. (2007). Napoleon Bonaparte, Political Prodigy. History Compass, 5(4), 1382-1398. https://doi.org/10.1111/j.14780542.2007.00451.x

Chapman, C. (2014). Théorie des couleurs, 1 : Signification de la couleur. Repéré à l'adresse https://lacascade.io/theorie-des-couleurs-1signification-de-la-couleur/

Cillessen, W., \& Reichardt, R. (2010). Matières scatologiques dans la caricature politique, de la Réforme à la Révolution. Annales historiques de la Révolution française, (361), 13-32. https://doi.org/10.4000/ahrf.11669

de Baecque, A. (1997). Daumier et les parlementaires de 1830 à 1875 ; Daumier et les gens de justice. Annales. Histoire, Sciences Sociales, 52(3), 531-533.

Duprat, A. (1999). Histoire de France par la caricature. Paris: Larousse.

Duprat, A. (2002). Les rois de papier: la caricature de Henri III à Louis XVI. Paris: Belin.

Dupuy, P. (2000). La caricature anglaise face à la France en Révolution (17891802). Dix-huitième Siècle, 32(1), 307-320.

https://doi.org/10.3406/dhs.2000.235 7

Dupuy, P. (2008). La violence française révolutionnaire au crible de la caricature anglaise (1789-1799). Repéré http://www.caricaturesetcaricature.c om/article-36188746.html

Huguenaud, K. (2002). Chapeau de Napoléon. Repéré à https://www.napoleon.org/histoiredes-2-empires/objets/chapeau-denapoleon/

Joly, M. (1994). Introduction à l'analyse de l'image. Paris: Nathan. 
Laurent, J.-M. (2015). Histoire de la caricature en France. Repéré à http://www.histoire-pourtous.fr/histoire-de-france/5014histoire-de-la-caricature-enfrance.html

Les couleurs. (s. d.). Repéré à http://www.bmigueret.fr/images/stories/couleurs_m ai_2013.pdf

Les symboles de la Révolution - La Révolution Française. (s. d.). Repéré à

https://sites.google.com/site/eterevol utionnaire1789/1-art-dans-larevolution/a-les-symboles-de-larevolution

Littlehale, K. (2017). King Lear Résumé, Personnages $\mathcal{E}$ Conflits | Héro Tragique. Repéré à http://www.storyboardthat.com/fr/t eacher-guide/le-roi-lear-parwilliam-shakespeare

Meister, H. (1993). Le discours de la caricature politique. Mots, 34(1), 101-106.

https://doi.org/10.3406/mots.1993.17 78

No Fear Shakespeare: King Lear: Act 3, Scene 4, Page 4. (s. d.). Repéré à http://nfs.sparknotes.com/lear/page 164.html

Oesterreicher-Mollwo, M. (Éd.). (1992). Petit dictionnaire des symboles. M. Broze \& P. Talon (Trad.). Turnhout, Belgique: Brepols.

Ouvrand, R. (2007). Napoléon à travers les caricatures 1799-1806 (1). Repéré à http://www.caricaturesetcaricature.c om/article-15673487.html

Pasquinelli, B. (2006). Le geste et l'expression. (C. Mulkai, Trad.). Paris: Hazan.

Porterfield, T. (2014). James Gillray, le mariage et le fonctionnement de la caricature. In S. Le Men (Éd.), L'art de la caricature (p.191-209). Nanterre: Presses universitaires de Paris
Nanterre. Repéré à

http://books.openedition.org/pupo/2 231

Rapelli, P. (2005). Symboles du pouvoir. (C. Garnier, Trad.). Paris: Hazan.

Reflexion sur la notion de vide dans la peinture. (s.d.). Repéré à http://www.daojia.fr/art/reflexionsur-la-notion-de-vide-dans-lapeinture

Reshef, O. (1984). Guerre, mythes et caricature: au berceau d'une mentalité française. Paris: Pr. de la Fondation nationale des sciences politiques.

Salles, D., \& Eymard, M. (s.d.). La caricature et le dessin de presse. Repéré à https://www.reseau-canope.fr/jedessine/la-caricature-et-le-dessinde-presse.html

Simonnet, D. (2004). 5. Le jaune: tous les attributs de l'infamie! Repéré à https://www.lexpress.fr/styles/5-lejaune-tous-les-attributs-de-1infamie_488943.html

SparkNotes: King Lear: Themes, Motifs \& Symbols. (s. d.). Repéré à http://www.sparknotes.com/shakes peare/lear/themes.html

The Genius of France Nursing Her Darling. (s.d.). Repéré à https://repository.library.brown.edu /studio/item/bdr:232470/

Wacheux, M. L. (2000). Naissance d'une icône ou les mésaventures caricaturales de «Little Boney» en Angleterre en 1803. Sociétés $\mathcal{E}$ Représentations, (2), 135-144. https://doi.org/10.3917/sr.010.0135

Walthall, B. (2016, mai 21). King Lear: How many wits does it take to... Repéré à https://thebillshakespeareproject.co m/2016/05/king-lear-many-witstake/

Woods, G. (2016). King Lear: madness, the fool and poor Tom. Repéré à https://www.bl.uk/shakespeare/artic les/king-lear-madness-the-fool-andpoor-tom 